\title{
Si rinnova il sito web del Gruppo di Studio di Dialisi Peritoneale
}

\author{
Roberto Russo \\ U.O. di Nefrologia Universitaria, Azienda Universitaria Consorziale Policlinico, Bari
}

\begin{abstract}
RENOVATION OF THE WEBSITE OF THE STUDY GROUP ON PERITONEAL DIALYSIS
Abstract. The new website of the Study Group on Peritoneal Dialysis (GdSDP) is characterized by the graphics, the multimedia contents, and eight subject areas. The innovations are the areas dedicated to Pediatrics, nurses, and e-learning (FAD) for obtaining CME credits. The chief editor is the coordinator of the GdSDP that relies on the contributions of the managers in the sector.
\end{abstract}

Key words: Peritoneal dialysis, Website, Multimedia contents, Nephromeet

Conflict of interest: None.

Financial support: None.

Ricevuto: 28 Novembre, 2013; Accettato: 2 Dicembre, 2013

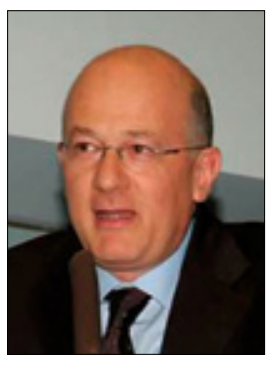

Roberto Russo
Il Gruppo di Studio di Dialisi Peritoneale (GdSDP) ha un nuovo sito web. Del vecchio sito rimane solo l'indirizzo www. dialisiperitoneale.org. Le novità riguardano la veste grafica, le modalità di gestione dei contenuti multimediali, l'organizzazione editoriale e le aree tematiche. Il GdSDP è il primo Gruppo di Studio della SIN che si dota di un sito web innovativo per l'organizzazione e la gestione dei contenuti. Il sito si collega, per quanto riguarda gli argomenti "generalisti", a una piattaforma web collaudata e interattiva come Nephro$M E E T$, che raccoglie le conoscenze nefrologiche sotto forma di documenti multimediali, con un sistema di interscambio di contenuti tipo "hub and spoke".

La modalità di gestione dei contenuti multimediali del nuovo sito utilizza l'area nefrologica di NephroMEET pur mantenendo la propria identità e il proprio ambito. L'accesso al sito avviene attraverso un login unico con NephroMEET e i contenuti pubblicati sul sito possono essere condivisi nell'intera area nefrologica, nel caso siano classificati come generali ( $p$. es., best practice, Linee Guida), mentre sono consultabili solo all'interno dell'area GdSDP nel caso siano classificati come locali e, cioè, strettamente correlati alla dialisi peritoneale (procedure, documenti, normative regionali, ecc.).

Il login di accesso al sito individua varie tipologie di utenti, come i soci SIN, i soci SIN iscritti al GdSDP e gli ospiti (infermieri, medici non nefrologi, pazienti, ecc.). Ogni categoria di utenti del sito, a seconda della scheda anagrafica compilata per l'iscrizione, potrà accedere a contenuti del sito diversificati; i soci SIN iscritti al GdSDP potranno accedere a tutti i contenuti disponibili. Previo login, ogni utente potrà accedere all'elenco degli utenti del sito, registrati nell'ambito della categoria alla quale appartengono, con la possibilità di accedere all'e-mail di ognuno. Infine, l'amministratore del sito potrà avere informazioni sul numero di accessi differenziati per tipologia di utente.

L'architettura del sito e la veste grafica sono cambiate nella speranza che la homepage attuale risulti organizzata meglio e più intuitiva (Fig. 1). Le aree tematiche sono otto: verbali e Comitato, censimento, audit, basic PD, documentale (lavori scientifici e normative regionali e nazionali sulla DP), studi, area pediatrica e area infermieristica. Nella homepage sono presenti, inoltre, un banner per le news, un banner che riporta ai contenuti multimediali dei precedenti convegni del GdSDP, un banner che richiama al prossimo convegno del GdSDP e, infine, un banner con un link alla consultazione dei contenuti e dei documenti necessari per la formazione ECM a distanza (FAD). Infine, nella homepage, sono presenti collegamenti con gli argomenti classificati come generali (best practice), che sono consultabili sia dal sito web del GdSDP che dal sito NephroMEET. Ogni area tematica che presenta documenti o contributi pubblicati di recente è contraddistinta da un banner "new", per un riconoscimento e una consultazione di quanto pubblicato più rapidi.

Il Responsabile Editoriale del sito è il coordinatore del GdSDP in carica, mentre ognuna delle aree tematiche è affidata a un Comitato Editoriale, con un Responsabile, che ha la funzione di aggiornarle costantemente. Tra le aree tematiche, oltre a 


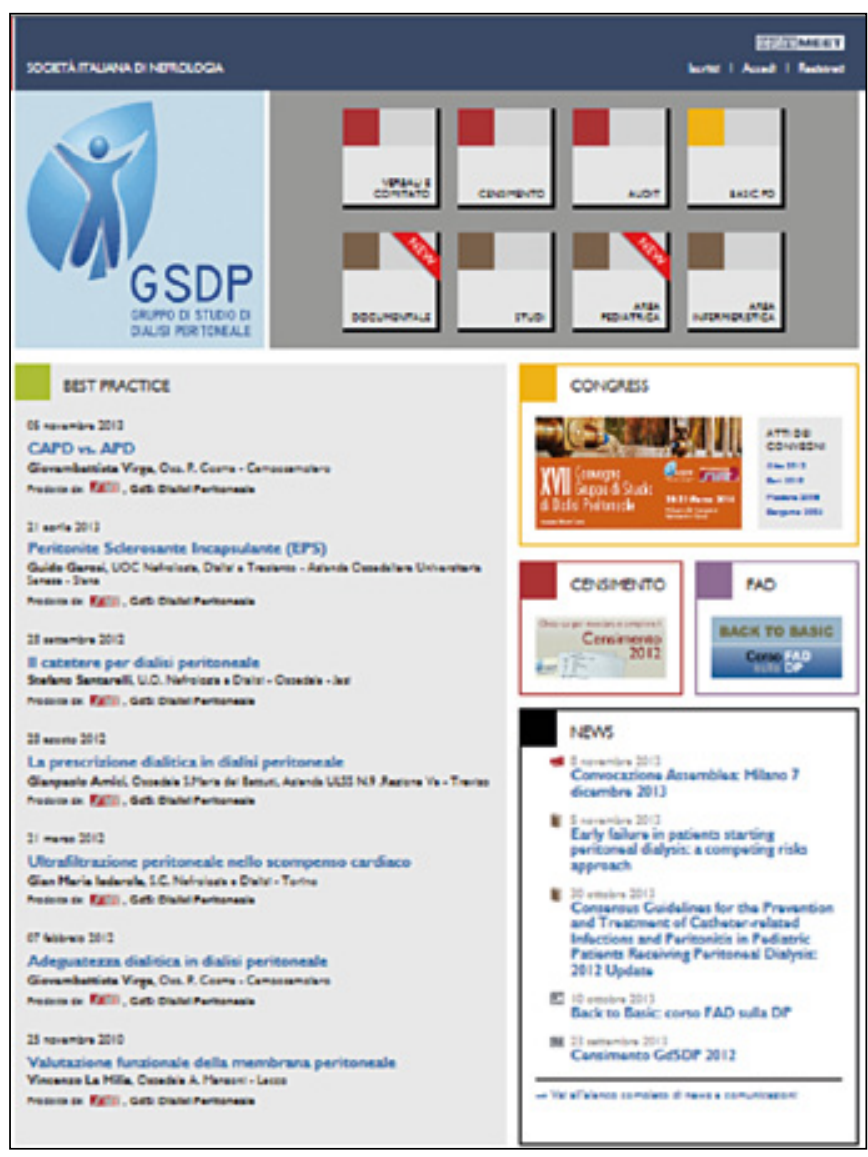

Fig. 1 - Homepage del sito del Gruppo di Studio di Dialisi Peritoneale.

quelle relative alla raccolta e alla pubblicazione dei verbali di Assemblea e del Comitato Scientifico del GdSDP, del censimento con la possibilità di accedere alla scheda raccolta dati di quello in corso e dei risultati dei censimenti precedenti, sono presenti l'area dedicata all'audit, che pubblica il materiale degli incontri tenuti nelle varie Regioni, e quella dedicata agli studi e ai protocolli promossi dal GdSDP. L'area documentale comprende una sezione dedicata al commento di articoli scientifici particolarmente interessanti sulla DP con aggiornamenti mensili e una sezione che raccoglie tutte le normative nazionali e regionali pubblicate sulla DP (incentivazioni eco- nomiche, promozione, piani sanitari, ecc.), ordinate per Regioni. Il nuovo sito, infine, è dotato di altre tre nuove aree: la basic PD con contenuti educazionali (argomenti sulla DP con diapositive commentate) e una FAD per crediti ECM, l'area pediatrica e quella infermieristica, che raccolgono contributi provenienti da due realtà così importanti e che completano l'aggiornamento clinico e professionale del nefrologo che si interessa di DP.

Il sito è attivo dallo scorso Settembre e, nelle intenzioni del Comitato Scientifico del GdSDP, è aperto alla collaborazione di tutti quelli che vorranno arricchirlo di contenuti.

\section{Riassunto}

Il nuovo sito web del Gruppo di Studio di Dialisi Peritoneale (GdSDP) si caratterizza per la veste grafica e le modalità di gestione dei contenuti multimediali e per otto aree tematiche. Una novità è rappresentata dalle aree dedicate alla pediatria, agli infermieri e alla formazione a distanza per il rilascio di crediti ECM. Il Responsabile Editoriale è il coordinatore del GdSDP in carica, che si avvale del contributo dei Responsabili d'area.

Parole chiave: Dialisi peritoneale, Sito web, Contenuti multimediali, NephroMEET

Dichiarazione di conflitto di interessi: L'Autore dichiara di non avere conflitto di interessi.

Contributi economici agli Autori: L'Autore dichiara di non aver ricevuto sponsorizzazioni economiche per la preparazione dell'articolo.

Indirizzo degli Autori:

Dr. Roberto Russo

U.O. di Nefrologia

Azienda Ospedaliero-Universitaria

Consorziale Policlinico di Bari

Piazza G. Cesare 11

70124 Bari

roberto.russo@policlinico.ba.it 